\title{
Computer analysis of alternative splicing events by RNA-seq data in brain cells
}

\author{
V.N. Babenko ${ }^{1}$, N.V. Gubanova ${ }^{1}$, A.V. Tsukanov ${ }^{1}$, S.S. Kovalev², A.O. Bragin ${ }^{1}$, \\ G.V. Vasiliev ${ }^{1}$, Yu.L. Orlov ${ }^{1,2 *}$ \\ ${ }^{1}$ Institute of Cytology and Genetics SB RAS, Novosibirsk, Russia \\ ${ }^{2}$ Novosibirsk State Medical University, Novosibirsk, Russia \\ ${ }^{3}$ Novosibirsk State University, Novosibirsk, Russia \\ *e-mail:orlov@bionet.nsc.ru
}

Key words: transcriptome, glioblastoma, alternative splicing, differential splicing, cancer stem cells

\begin{abstract}
Motivation and Aim: Alternative splicing is a critical mechanism for expanding regulatory and functional diversity from a limited number of genes. It is particularly complex in the mammalian brain [1]. Glioblastoma is the most common and aggressive type of primary brain tumor, accounting for $80 \%$ of malignant astrocytomas. Therefore, it is critical that the genetic pathways underlying the development of this type of cancer are defined.

Methods and Algorithms: The gene expression profiles of glioblastoma were obtained on cell culture samples of primary glioblastoma isolated and processed for RNA extraction. Transciptome profiling of normal brain samples and glioblastoma were done by Illumina sequencing. We used set of computer tools applied recently to analysis of gene expression in laboratory animals to study differential splicing events. To analyze alternative splicing events in the transcriptomics data MATS (multivariate analysis of transcript splicing) and rMATS (replicate MATS) were used as tools.
\end{abstract}

Results: We identified gene loci with highly significant differential isoforms expression. The major GO entries for alternatively spliced genes were cytoskeleton and intracellular (cytoplasmic) related genes. We found also genes of nuclear pore complex as differentially expressed in NGB cell culture sample. During the analysis of differential splicing events we found significant differences in splicing of three cancer associated genes, in particular: APP (amyloid beta precursor protein), CASC4 (cancer susceptibility candidate 4) and TP53.

Conclusion: Multiple alternative splicing transcripts have been identified as progression markers, including generalized splicing abnormalities and tumor- and stage-specific events.

Acknowledgements: The work was supported by RFBR, ICG SB RAS budget project 0324-2018-0017.

\section{References}

1. Babenko V.N. et al. (2017) Computer analysis of glioma transcriptome profiling: alternative splicing events. J. Integrative Bioinformatics. 14(3). pii: /j/jib.2017.14.issue-3/jib-2017-0022/jib-2017-0022.xml. 